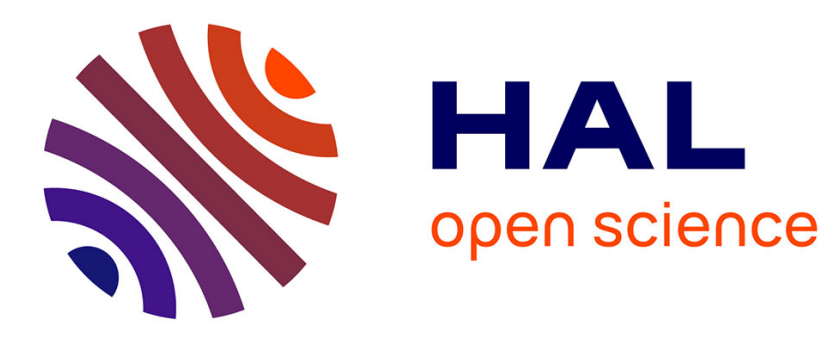

\title{
Demonstrator Detection System for the Active Target and Time Projection Chamber (ACTAR TPC) project
}

T. Roger, J. Pancin, G.F. Grinyer, B. Mauss, A.T. Laffoley, P. Rosier, H. Alvarez-Pol, M. Babo, B. Blank, M. Caamaño, et al.

\section{- To cite this version:}

T. Roger, J. Pancin, G.F. Grinyer, B. Mauss, A.T. Laffoley, et al.. Demonstrator Detection System for the Active Target and Time Projection Chamber (ACTAR TPC) project. Nucl.Instrum.Meth.A, 2018, 895, pp.126-134. 10.1016/j.nima.2018.04.003 . hal-01781925

\section{HAL Id: hal-01781925 \\ https://hal.science/hal-01781925}

Submitted on 5 May 2018

HAL is a multi-disciplinary open access archive for the deposit and dissemination of scientific research documents, whether they are published or not. The documents may come from teaching and research institutions in France or abroad, or from public or private research centers.
L'archive ouverte pluridisciplinaire HAL, est destinée au dépôt et à la diffusion de documents scientifiques de niveau recherche, publiés ou non, émanant des établissements d'enseignement et de recherche français ou étrangers, des laboratoires publics ou privés. 
The design, realization and operation of a prototype or "demonstrator" version of an active target and time projection chamber (ACTAR TPC) for experiments in nuclear physics is presented in detail. The heart of the detection system features a MICROMEGAS gas amplifier coupled to a high-density pixelated pad plane with square pad sizes of $2 \times 2 \mathrm{~mm}^{2}$. The detector has been thoroughly tested with several different gas mixtures over a wide range of pressures and using a variety of sources of ionizing radiation including laser light, an $\alpha$-particle source and heavy-ion beams of ${ }^{24} \mathrm{Mg}$ and ${ }^{58} \mathrm{Ni}$ accelerated to energies of $4.0 \mathrm{MeV} / \mathrm{u}$. Results from these tests and characterization of the detector response over a wide range of operating conditions will be described. These developments have served as the basis for the design of a larger detection system that is presently under construction. 
Keywords: Active Target, Time Projection Chamber, Micromegas, Nuclear Physics

\section{Introduction}

The use of active targets and time projection chambers (TPCs) for experiments in nuclear physics can be traced back nearly 30 years. Historically, their intrinsic luminosity, three-dimensional tracking and particleidentification capabilities and relatively low-energy detection thresholds were some of the main characteristics where these types of detection systems offered a more attractive option when compared to conventional experimental techniques.Examples include IKAR at GSI [1], the MSTPC at RIKEN [2], the Maya active target at GANIL [3], the University of Warsaw optical TPC [4] and the CENBG TPC [5]. A detailed review of existing active targets can be found in Ref.[6]. While extremely successful, many of these previousgeneration detectors were each faced with a number of experimental challenges and limitations that were a result of the technology available at the time of their construction.

With recent technological developments in micro-pattern gaseous detectors (MPGDs) [7 10], connectors and cables to achieve higher-density pointto-point contacts and electronics and data-acquisition systems that feature front-end data processing with high data throughput, present-day active targets and time projection chambers can achieve significantly higher channel densities than could previously be contemplated. Combined with ongoing developments in the production and acceleration of short-lived beams of rare isotopes at radioactive ion-beam facilities worldwide, the demand for active targets and time projection chambers in the nuclear physics community has increased dramatically [1].

The active target and time projection chamber (ACTAR TPC) is an ambitious detector development project whose goal is to develop a state-ofthe-art detection system that consists of one (or possibly several) MPGDs coupled to a pixelated pad plane consisting of square pads with a pitch of $2 \times 2 \mathrm{~mm}^{2}$. Both the overall channel density of $25 \mathrm{pads} / \mathrm{cm}^{2}$ and the total

Email addresses: roger@ganil.fr (T. Roger), pancin@ganil.fr (J. Pancin)

${ }^{1}$ Present address: Riken Nishina Center, 2-1 Hirosawa, Wako, Saitama, 351-0198, Japan 
number of channels (16384) are, to the best of our knowledge, the highest that have been achieved by any detector in nuclear physics to date. In the present article, the design construction and operation of a 2048-channel prototype or "demonstrator" version of the ACTAR TPC design is described in detail. Calibration methods used to characterize the detector as well as the results of radioactive source, laser and in-beam tests will be presented.

\section{ACTAR TPC Demonstrator Design}

\subsection{General layout}

The main goal of the ACTAR TPC demonstrator was to develop a fully functional prototype detector that would be used to assess the feasibility and robustness for all aspects related to its mechanical design. Some of the most crucial components that required verification before moving towards the final design included the mechanics of the pad plane, the design of the electrostatic field cage, the choice of connectors and cables, and the electronics integration. The ACTAR TPC demonstrator has a total of 2048 channels, which is 8 times smaller than the full-size detector that is presently under construction.

The ACTAR TPC demonstrator was designed at the Institute de Physique Nucléaire d'Orsay (IPNO). The base geometry of the detector consists of an aluminum inner skeleton whose central volume is $300 \times 250 \times 210 \mathrm{~mm}^{3}$. The detector is encased with 6 removable side flanges (top, bottom and 4 sides) as shown in Figure 1. One of the side flanges was designed with a $12 \mathrm{~mm}$ diameter circular entrance window to allow the detector to be coupled to a beam line. A $6 \mu \mathrm{m}$ thick Mylar foil serves as the sole interface between the beam line (vacuum, $10^{-6} \mathrm{mbar}$ ) and the gas volume of the detector itself that is typically operated with pressures ranging from 100 to 1000 mbar. The remaining 3 side-flanges are dedicated to support optional ancillary detectors. Gas flow into and out of the chamber uses two connectors located on opposite side flanges. The top flange (cathode) was designed to support vertically adjustable radioactive sources that can be located in one of two possible positions. The cathode voltage is supplied through a safe high-voltage (SHV) connector located on this flange. The bottom flange (the pad plane) houses the core of the TPC detection system. The chamber was designed to sustain 1 atm differential pressure, so that the active target can run from $\sim 0$ bar to $\sim 2$ bar, if equipped with proper O-rings.

The rectangular pad plane consists of an active area of $64 \times 128 \mathrm{~mm}^{2}$. Individual square copper pads with a pitch of $2 \mathrm{~mm}$ and separated by $80 \mu \mathrm{m}$ 
tile the surface of the pad plane for a total $32 \times 64$ pads or 2048 total pixels. A MICROMEGAS amplification system [7] was deposited on top of the pad plane using the bulk technique [8]. A wire field cage surrounds the pad plane at a distance of $5 \mathrm{~mm}$ from the edge of the micromesh and extends to a vertical height of $170 \mathrm{~mm}$. The role of the field cage is to maintain a homogeneous vertical electric field across the entire drift region above the pad plane. The precise geometry of the field cage will be described below in section 3.2. Both the pad plane and the field cage are fixed onto the bottom flange, as shown in Fig. 1. An additional 3 SHV connectors situated on this flange are used to supply the voltage for the MICROMEGAS and the field cage. A more detailed view of the mechanical design of the pad plane is presented in Fig. 2 .

\subsection{Micromegas and pad plane}

Ionization electrons produced in the gas volume will be guided to the bottom of the detector under a uniform electric field. Amplification of the electron signals is performed using a MICROMEGAS system situated at the bottom of the field cage. The MICROMEGAS consists of a 45/18 stainless-steel woven micromesh laminated on $256 \mu \mathrm{m}$ high insulating pillars. The amplification gap between the pad plane and the micromesh is approximately $220 \mu \mathrm{m}$ after the lamination process. The MICROMEGAS bulk was manufactured by the CERN PCB workshop. This particular gap thickness is relatively large when compared to standard MICROMEGAS detectors. This choice was motivated by results obtained in our previous study that demonstrated that the larger gap was better suited to a wider range of operating gas pressures including low pressures of $\sim 100$ mbar [12]. Typical operating voltages of the MICROMEGAS range from $-200 \mathrm{~V}$ to $-1000 \mathrm{~V}$ depending on the type of gas, pressure and desired gain. In order to minimize the capacitance of the pixel routing and the total number of layers in the PCB pad plane, the connectors on the exterior side of the pad plane were chosen so that they occupy the same surface area as the active surface on the interior side. The pad plane serves as the primary interface between the gas volume of the detector and the outside (atmospheric pressure). To clean the chamber before filling it with gas, it must be pumped down to primary vacuum. The pad plane must therefore be able to sustain $\sim 1$ atm differential pressure with minimal mechanical deformation. To further reinforce the mechanical rigidity, the PCB pad plane was then glued and screwed onto a $15 \mathrm{~mm}$ thick aluminum plate. Small openings of $30 \times 7 \mathrm{~mm}^{2}$ were machined into the plate to allow the connectors on the PCB to pass through it, as shown in Fig. 2. The $2 \times 48$-channel 


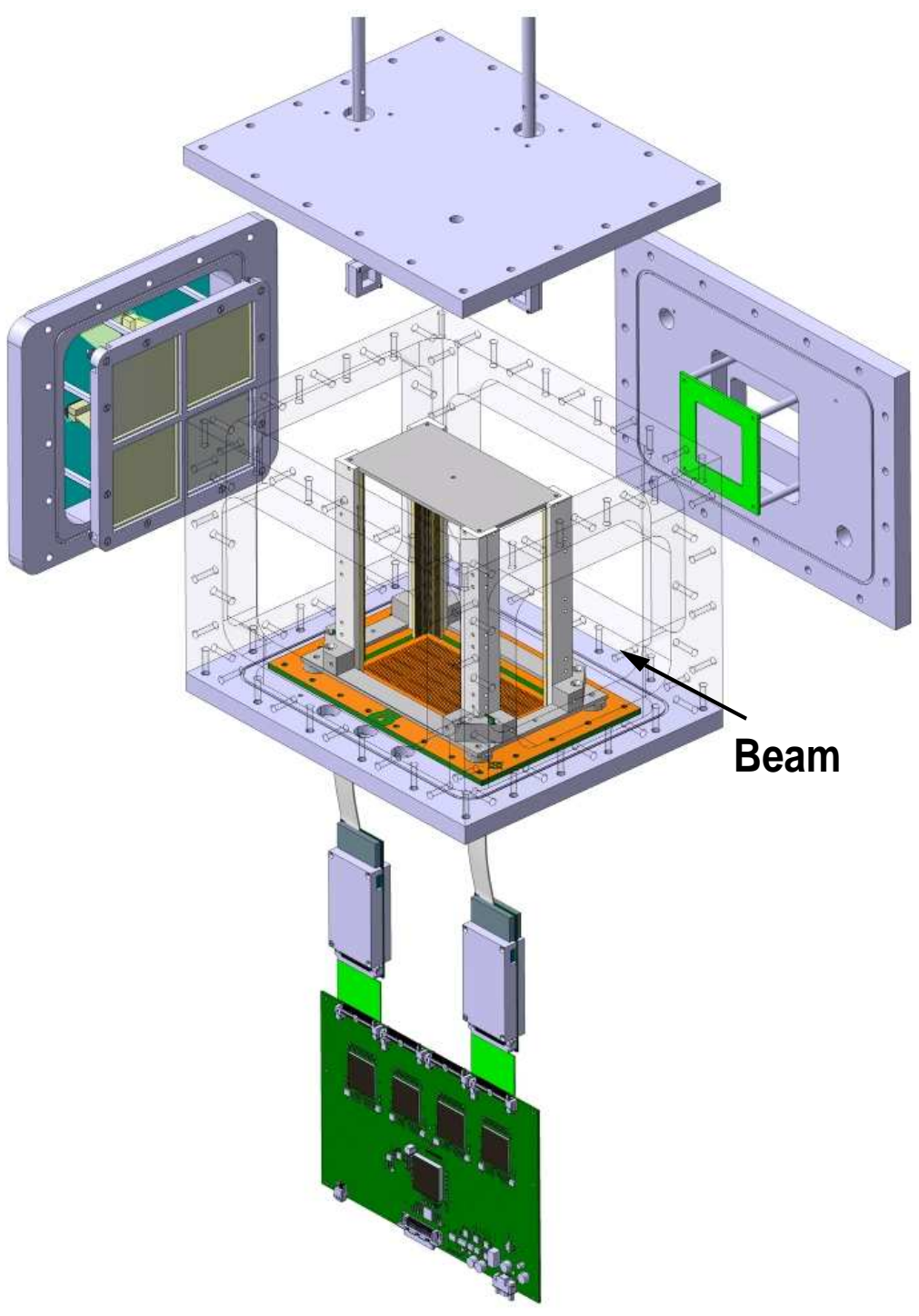

Figure 1: (Color online) 3D drawing of the ACTAR TPC demonstrator. The downstream (left) and the right side flanges are shown equipped with four and one double sided stripped silicon strip detectors, respectively. The top flange holds two retractable and vertically adjustable radioactive source arms. The four field cage pillars and the cathode above them are mounted on the pad plane (bottom flange). Outside the chamber and situated below the pad plane, the connection of a single front end electronics card via two sparkprotection circuits is shown. A total of 8 cards are required to process the 2048 channels of the demonstrator. 


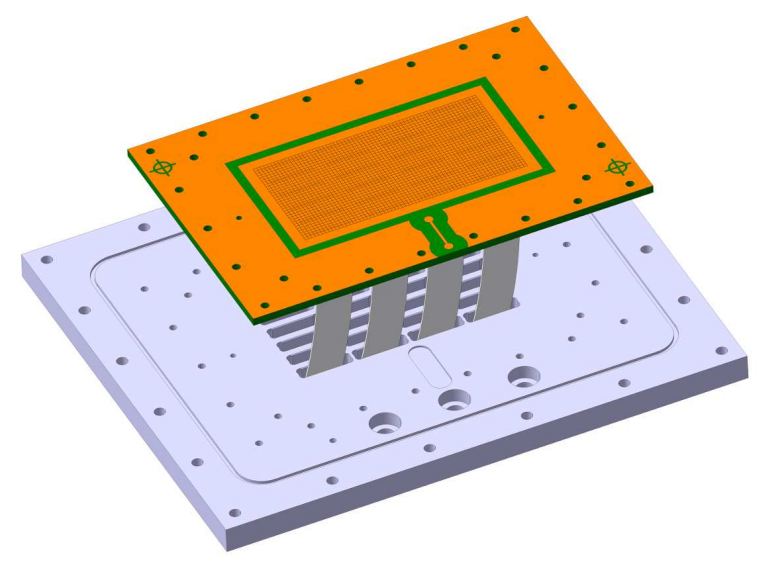

Figure 2: (Color online) Drawing of the pad plane and aluminum frame assembly.

SAMTEC connectors have a pitch of $0.8 \mathrm{~mm}$ and were wave soldered onto the pad plane before the MICROMEGAS were assembled above. The final pad plane is a 4-layer PCB with a thickness of $4 \mathrm{~mm}$. Mechanical simulations were performed to confirm that the deformation of the entire assembly (pad plane PCB and the aluminum frame) was less than $100 \mu \mathrm{m}$ at a differential pressure of $1 \mathrm{~atm}$.

\subsection{Electronics and data-acquisition system}

Data from the 2048 channels of the ACTAR TPC demonstrator are processed and stored using the General Electronics for TPCs (GET) system [13]. Between the pads and the electronics a set of 32 protection circuits with 64 channels each were designed to protect the front end from highly saturating signals that could arise from sparks. The spark-protection circuits were connected to the pad plane using $12 \mathrm{~cm}$ Teflon NEXAN coaxial cables with $85 \mathrm{pF} / \mathrm{m}$ linear capacitance.

The GET system is a comprehensive and generic digital electronics and data-acquisition system that was designed for nuclear physics instrumentation with up to 30000 channels. A full description of the GET system is provided in Ref. [13]. A brief summary is provided here for some of the features that are of relevance to the ACTAR TPC demonstrator. The front-end electronics consists of a custom designed and versatile 64-channel Application Specific Integrated Circuit (ASIC) chip called "ASIC for GET" or AGET. The AGET provides, for each channel, a charge sensitive pre-amplifier with 
adjustable gain (dynamic ranges of $120 \mathrm{fC}, 240 \mathrm{fC}, 1 \mathrm{pC}$ and $10 \mathrm{pC}$ ), an analogue filter (shaper) with a peaking time ranging from 70 to $1014 \mathrm{~ns}$, a leading edge discriminator and a 512-cell switch capacitor array to continuously sample the signal with a maximum frequency of $100 \mathrm{MHz}$. Four AGET chips (4×64 or 256 channels) each followed by a 12-bit Analogue to Digital Converter $(\mathrm{ADC})$ are integrated onto a front-end board called AsAd (ASIC and ADC). A schematic of one AsAd card with 4 AGET chips is shown in Fig 1. Digitized data from up to 4 AsAd cards $(4 \times 256$ or 1024 channels $)$ are collected and processed by a concentration board (CoBo). The CoBo cards are compatible with the Micro Telecommunications Computing Architecture ( $\mu$ TCA) and are operated in a Vadatech VT893 $\mu$ TCA chassis. A total of $2 \mathrm{CoBo}$ cards are required to read the 2048-channels of the demonstrator pad plane. Synchronization and distribution of the $100 \mathrm{MHz}$ global master clock through the $\mu$ TCA backplane is performed using the Multiplicity Trigger and Time (MuTanT) module. The MuTanT also provides multi-level triggering capabilities. System master trigger decisions can be derived from external sources (L0), overall pad multiplicity (L1) or through a user-specific hit-pattern algorithm (L2).

Communication between the $\mu \mathrm{TCA}$ chassis and the data-acquisition computer is achieved through an optical fibre from the 10 GbE network switch on the $\mu$ TCA Carrier Hub (MCH). The $\mathrm{MCH}$ is a commercially available module that operates in a dedicated slot of the $\mu$ TCA chassis. Detailed technical descriptions of the $\mu \mathrm{TCA}$ architecture and the MuTanT module are provided in Ref. [14]. The data-acquisition computer is a DELL PowerEdge R420 server with dual 6-core/12-thread Xeon E5-2430 2.2 GHz, 32 GB of random-access memory (RAM) and $8 \mathrm{~TB}$ of total disk space. The data acquisition software running on LINUX CentOS was developed for the GET system and consists of 3 main subsystems. The electronics control core (ECC) is used to load the particular hardware configurations and monitor the electronics boards. The data flow subsystem is based on the existing NARVAL modular data-acquisition framework [15] that contains specific processes that are used for data collection, event building and merging, online data processing and filtering, and data storage. The run control subsystem is a user-friendly graphical user interface that incorporates the ECC and data flow subsystems to provide global control and monitor the status of the entire system.

The configuration of the GET electronics is performed using a java-based Graphical User Interface developed at GANIL. This software provides an easy way of configuring the electronics parameters such as the gain, threshold 
and peaking time of the individual channels as well as setting the triggering conditions.

\section{Operation of the ACTAR TPC Demonstrator}

\subsection{Electronics and MICROMEGAS calibration}

Amplified signals from the charge pre-amplifiers of the GET system exhibit channel-by-channel gain fluctuations that are typically on the order of $\sim 10 \%$. A relative gain alignment of the individual channels must therefore be applied before performing any detailed analysis. Aligning the individual channels requires a two-step process. The first is to send an external pulser to the mesh of the MICROMEGAS so that a charge can be injected in all channels. While this step is relatively straightforward, the amount of charge deduced at the channel level will depend upon both the gain of the pre-amplifier for that specific channel and the size of the gap between the mesh and the pad at that particular location. To disentangle the relative contributions from both of these, a second step is then required to deduce the height of the mesh-pad gap on a pad-by-pad basis. A measurement of the gap homogeneity for the MICROMEGAS detector used in the ACTAR TPC demonstrator was performed using the method described in Ref. [16]. A collimated ${ }^{55} \mathrm{Fe} \mathrm{X}$ ray source mounted on an automated 2-dimensional scanning table was used to correlate the measured response of the mesh with the precise position of the source. The relative gap of the micromesh was then reconstructed using Magboltz [17] and converted into an effective gain for any combination of gas species or amplification voltage used.

A scan of the pad plane was performed using an $\operatorname{Ar}(97 \%)+\mathrm{iC}_{4} \mathrm{H}_{10}(3 \%)$ gas mixture at a pressure of 1021 mbar. The resulting gain map and deduced micromesh-pad gap values are presented in Fig. 3. The gain fluctuation measured was of the order of $10 \%$. The deduced gap height across the entire surface was found to be homogeneous to within $\pm 1 \%$. Such small variations are negligible when compared to the channel-by-channel gain fluctuations associated with the electronics. Injecting an external pulser on the mesh as a means to calibrate the gain fluctuations of the electronic channels is therefore fully justified for a relatively homogeneous MICROMEGAS gap.

\subsection{Field cage and drift field homogeneity}

The homogeneity of the electric field is essential for accurately reconstructing particle trajectories through the drift volume. The ideal field cage 


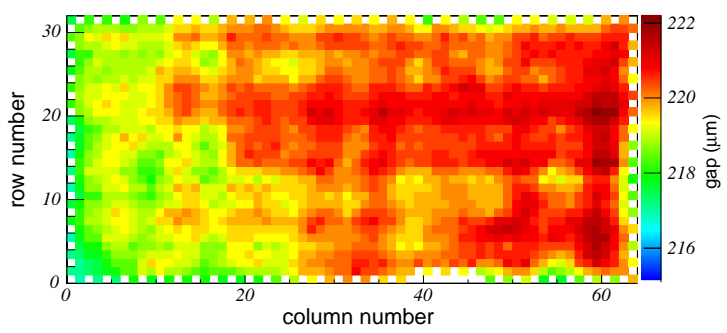

Figure 3: (Color Online) MICROMEGAS gap homogeneity map deduced with an $\operatorname{Ar}(97 \%)+\mathrm{iC}_{4} \mathrm{H}_{10}(3 \%)$ gas mixture at 1021 mbar. A reference gap of $220 \mu \mathrm{m}$ was chosen for the Magboltz calculations.

is one that would maintain a static and homogeneous electric field that is completely perpendicular to the surface of the micromesh (vertical in the case of ACTAR TPC) with absolutely no components acting in the parallel (horizontal) direction. In practice, small parallel fields can be tolerated provided that their magnitude, relative to the perpendicular electric field, are less than the ratio of half the pad size $(1 \mathrm{~mm})$ to the drift height $(17 \mathrm{~cm})$. This upper limit of $0.6 \%$ for the field-cage tolerance is to ensure that the difference between the creation points of the ionization electrons and their corresponding mesh arrival points are smaller than 1 pad.

In many experiments, the reaction products will have sufficient energy to escape the drift volume. The field cage must therefore be transparent to allow these particles to pass through the cage so that they can be detected in a set of auxiliary detectors such as scintillators or silicon detectors. Special care must also be taken to ensure that the presence of these auxiliary detectors do not disturb the drift field. Following several tests and a detailed simulation study (described below), a double wire-plane field-cage design was chosen. This design, while more complex, outperformed a single wire-plane cage design in terms of overall electric field homogeneity. The field cage is connected to two separate power supplies, to fix the potentials on the top of the cathode and at the bottom, via a set of resistors to the ground. The value of the resistor depends on the voltage applied on both power supplies. It is of the order of $10 \mathrm{M} \Omega$. The mesh is polarized separately to be able to tune its voltage to a different value than for the field cage bottom, in order to compensate for side effects of the electric filed on the border of the pad plane, and to avoid the field cage current to be sent in the mesh.

The double wire-plane field cage designed for the ACTAR TPC demon- 
strator consists of an internal cage with 4 wire planes situated at a distance of $5 \mathrm{~mm}$ from the edge of the pad plane. The distances between the field cage and the interior walls of the chamber are $40 \mathrm{~mm}$ in the vertical direction (above the cathode) and about $70 \mathrm{~mm}$ on all four sides. The voltage between the cathode and the mesh of the MICROMEGAS is homogeneously degraded using 4.7 M $\Omega$ (1\%) surface-mounted resistors soldered between each wire. Each wire plane consists of 169 wires each with a diameter of $20 \mu \mathrm{m}$ and a $1 \mathrm{~mm}$ spacing. The second field cage surrounds the first and consists of 4 wire planes with 34 wires each. The wire diameter is also $20 \mu \mathrm{m}$ and the wire spacing is $5 \mathrm{~mm}$. The distance between the inner and outer wire planes is $10 \mathrm{~mm}$ on all sides and their total height (the height of the drift volume) is $170 \mathrm{~mm}$. With this design, the optical transparency is deduced to be nearly $98 \%$.

The majority of unwanted stray electric fields in the drift region arise, in our particular case, from electric fields that are generated between the field cage and an array of silicon detectors that were placed at a distance of $\sim 5.0 \mathrm{~cm}$ from the sides of the cage. Assuming that the cathode bias is $3500 \mathrm{~V}$, which corresponds to a drift electric field of approximately $200 \mathrm{~V} / \mathrm{cm}$ in the vertical direction, and that the $\mathrm{Si}$ detectors are at ground, then the maximum horizontal electric field will be at the cathode and will be on the order of $700 \mathrm{~V} / \mathrm{cm}$. The magnitude of this field decreases linearly with the drift height. As described above, the goal of the field cage is to suppress these fields from reaching the interior of the drift volume. Ideally this suppression factor should therefore be on the order of 600 in order to achieve the $0.6 \%$ design goal. A simple calculation of this suppression factor was performed using the formalism described in Ref. [18] for a single wire cage (with $1 \mathrm{~mm}$ wire pitch and $20 \mu \mathrm{m}$ diameter wires) and the resulting suppression factor of $\sim 90$ was found. This is in good agreement with estimates from electric field calculations using Garfield [19]. The Garfield calculations were then extended to estimate the overall suppression factor for the double wire-plane field-cage configuration using the geometry described above. The resulting suppression factor was determined to be $\sim 270$. The additional factor of 3 gained with the double wire field cage is therefore essential for reducing the transverse electric fields while maintaining a suitable optical transparency.

The homogeneity of the drift electric field was characterized using a collimated alpha source. Identical tests were performed using both a single field cage with a wire spacing of $1 \mathrm{~mm}$ and the double field cage (inner cage with $1 \mathrm{~mm}$ wire pitch surrounded by a second cage with $5 \mathrm{~mm}$ wire pitch). With 
the single cage, alpha-particle trajectories showed clear evidence for horizontal deformation. The amplitude of this deformation increased even further for particles emitted in the direction of the cathode where the transverse electric fields are largest. An example of a deformed trajectory is presented in Fig. (4a) for the single wire-plane field cage. In this event, the alpha particle was emitted from the left side of the figure and traverses the active volume of the TPC before being detected in one pixel of a double sided Si strip detector (DSSSD) placed outside and approximately $5 \mathrm{~cm}$ from the field cage. This particular pixel of the DSSSD was chosen because it was situated close to the cathode in the vertical direction and near the corner of the field cage in the horizontal. This event thus corresponds to one of the largest deformations observed.

A similar alpha-particle trajectory recorded using the same pixel of the DSSSD but with the double wire-plane field cage is shown in Fig. 4b). The overall horizontal deformation in the double cage is significantly reduced compared to the single wire-plane field cage. Again, this particular trajectory represents one of the most extreme cases. Considering all possible horizontal and vertical alpha-particle emission angles, the majority of events would be significantly less deformed.

\subsection{Laser tests}

The horizontal and vertical angular resolution with $2 \times 2 \mathrm{~mm}^{2}$ square pads was investigated in Ref. [12] where it was shown that the resolution was largely dominated by the straggling of the alpha particles in the gas. A new method, that relies on the use of an ultra-violet laser light, was designed and tested as a means to determine the intrinsic angular resolution of the detector in the absence of straggling. The titanium sapphire (Ti:Sa) laser employed was pumped by a $75 \mathrm{~W} 532 \mathrm{~nm}$ neodymium-doped yttrium aluminum garnet (Nd:YAG) laser (photonics industries CM57-532) frequency doubled at $10 \mathrm{kHz}$ repetition rate. After triple harmonic generation, the Ti:Sa laser produced $162 \mathrm{~mW}$ pulses of $140 \mathrm{~ns}$ duration at a wavelength of $259 \mathrm{~nm}$. These pulses were of sufficient energy to ionize aromatic hydrocarbons, present in the detector as impurities, via two-photon absorption. The laser light entered the detector through a quartz window that was mounted on one of the side flanges of the detector. At $259 \mathrm{~nm}$, the transmission of this window was measured using a photo sensor to be $\sim 98 \%$. The horizontal angle of the laser light was adjusted using a set of mirrors placed just before the entrance window to the detector. The active volume of the TPC was filled with 1 bar 

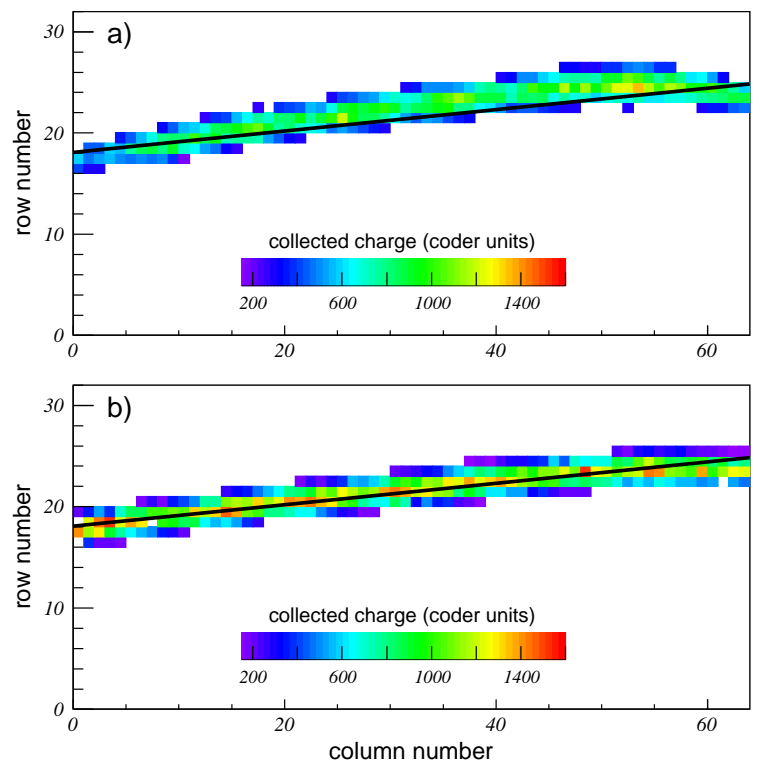

Figure 4: (Color online) Charge projection of an alpha-particle track in 40 mbar of isobutane, with a single wire-plane field cage (a) and with a double wire-plane field cage (b). In both figures, the cathode voltage was set to $-2500 \mathrm{~V}$ and the mesh voltage was $-400 \mathrm{~V}$. The trigger for the data acquisition was provided by one pixel (near the cathode or top of the drift field) of a double sided Si strip detector (DSSSD) located approximately $5 \mathrm{~cm}$ behind the field cage. In both figures, the black solid line represents the physical trajectory of the alpha particle from the collimated alpha source to the center of the pixel of the DSSSD.

of $\operatorname{Ar}(97 \%)+\mathrm{iC}_{4} \mathrm{H}_{10}(3 \%)$ gas mixture. The cathode voltage was $-2500 \mathrm{~V}$ and the mesh voltage was set to $-380 \mathrm{~V}$. The recorded charge projection for a typical laser pulse aimed at the corner of the field cage is presented in Fig.5 5 ). The curvature exhibited near the end of the trajectory is again due to deformation induced by the single wire-plane field cage that was described above in Sec. 3.2. The three-dimensional angle of the tracks was reconstructed using an algorithm derived from those presented in Ref. [20]. As the curvature arises from a systematic effect, a fit to the entire trajectory using a straight line will result in a systematic shift to the reconstructed angle. However the width of the angular distribution presented in Fig.5 not affected by this curvature. From Fig $5 \mathrm{~b}$ ), and neglecting the divergence of the laser beam, an angular resolution of $0.06^{\circ} \mathrm{FWHM}$ was achieved. This result indicates that, for the case of long trajectories, the angular resolution 
for tracking particles through the active target will be entirely limited by the straggling of the ions in the gas rather than geometrical effects associated with the size of the individual pixels.
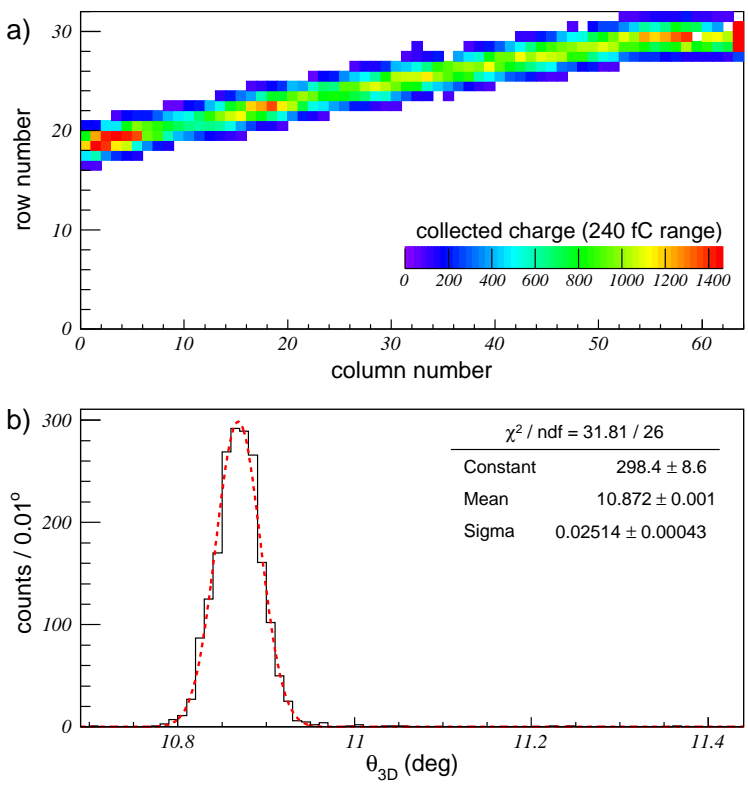

Figure 5: (Color Online) a) Recorded charge projection of a single $259 \mathrm{~nm}$ laser pulse in a gas mixture of $\operatorname{Ar}(97 \%)+\mathrm{iC}_{4} \mathrm{H}_{10}(3 \%)$ at 1 bar pressure. b) Three-dimensional angular resolution deduced from fitting many trajectories

\subsection{Alpha-particle source tests}

The energy resolution of the detection plane was further investigated using a ${ }^{239} \mathrm{Pu},{ }^{241} \mathrm{Am}$ and ${ }^{244} \mathrm{Cm}$ mixed alpha source placed $35 \mathrm{~mm}$ downstream the pad plane. The detector was filled with 148 mbar of pure isobutane, which is sufficient to fully stop the alpha particles inside the active volume. The voltages applied on the cathode and the micromesh were set to $-4000 \mathrm{~V}$ and $-400 \mathrm{~V}$, respectively. The total charge deposited by the alpha particles was obtained on an event-by-event basis by summing all of the individual charges collected on each pad. The individual charges were reconstructed using the procedures described in Ref. 21]. A typical total charge spectrum for the alpha source is presented in Fig. 6. This spectrum was obtained by restricting the analysis to horizontal angles between $\pm 1^{\circ}$ in order to minimize the effect of the dead zone between the source and the beginning of the pad plane. A 
fit to this spectrum using three Gaussian distributions yielded energy resolutions of $5.4 \%, 4.9 \%$ and $5.5 \%$ (FWHM) for alpha-particle energy deposit of $3.74 \mathrm{MeV}, 4.14 \mathrm{MeV}$ and $4.53 \mathrm{MeV}$, respectively. These results are encouraging when compared to the previous values of $5 \%, 4.5 \%$ and $6 \%$ (FWHM) that were obtained in Ref. [12] and that used a mixture of $\operatorname{Ar}(98 \%)+\mathrm{CF}_{4}(2 \%)$ at 1100 mbar gas pressure and a MICROMEGAS detector with a gap of $256 \mu \mathrm{m}$ coupled to a $2 \times 2 \mathrm{~mm}^{2}$ pad plane.

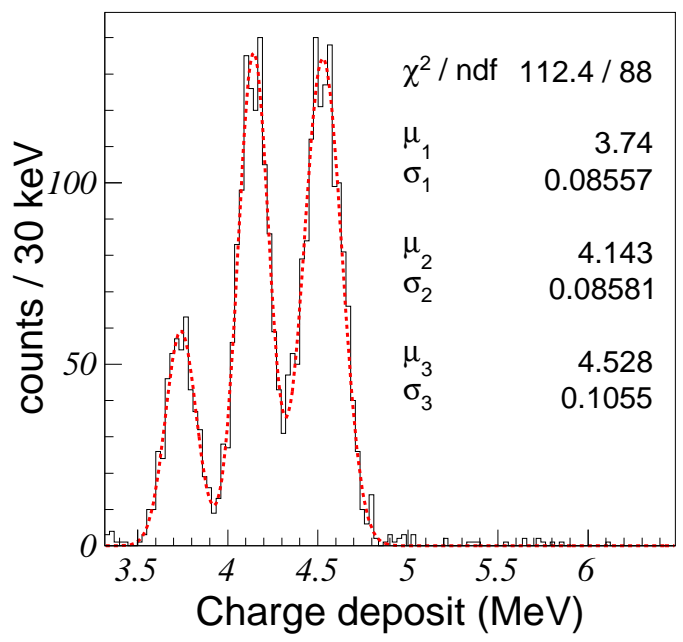

Figure 6: (Color Online) Total charge spectrum from a ${ }^{239} \mathrm{Pu}+{ }^{241} \mathrm{Am}+{ }^{244} \mathrm{Cm}$ mixed alpha source. The red dashed line corresponds to a fit to the histogram using 3 Gaussian distributions. The fit parameters are indicated in the inset. The fit parameters $\mathrm{A}, \sigma$, and $\mu$ correspond to the maximum peak height, the standard deviation, and the position of the centroid, respectively.

\subsection{Gain characterization measurements}

The highest energy alpha particle at $5.8 \mathrm{MeV}$ from ${ }^{244} \mathrm{Cm}$ decay was also used to characterize the gain of the MICROMEGAS amplification system for various gas mixtures at several different pressures. For each gas mixture and pressure, measurements were performed using several different mesh voltages. For each measurement, approximately 30 horizontal alpha trajectories were selected, and the average charge deposited was calculated on a pad-bypad basis. This averaged alpha-particle trajectory was then projected along its axis in order to obtain the average energy-loss profile. This profile is a 
convolution of the true energy loss of the alpha particles with a Gaussian distribution to describe the lateral straggling of the ionization electrons in the gas. Examples of several average energy-loss profiles that were measured in a $\mathrm{He}(90 \%)+\mathrm{iC}_{4} \mathrm{H}_{10}(10 \%)$ gas mixture at 700 mbar are presented in Fig:7a). At high mesh voltage, the gains of the charge pre-amplifiers were reduced to avoid saturating the electronic signals. The gain of the MICROMEGAS was then deduced through a comparison of the registered charge profile with a SRIM simulation [22] of the energy loss of the alpha particles in the gas convoluted with the lateral straggling of the electrons. The gain of the amplifer as a function of the applied mesh voltage is presented in Fig. 7b) for several different gas mixtures and pressures including $\mathrm{He}(90 \%)+\mathrm{iC}_{4} \mathrm{H}_{10}(10 \%)$ at 96 mbar and 700 mbar, $\mathrm{He}(95 \%)+\mathrm{iC}_{4} \mathrm{H}_{10}(5 \%)$ at 139 mbar and for pure $\mathrm{iC}_{4} \mathrm{H}_{10}$ at 98 mbar and 148 mbar. The gain curves of the low pressure $\mathrm{He}+$ $\mathrm{iC}_{4} \mathrm{H}_{10}$ mix exhibit a smaller slope than those of the other gases tested due to a saturation of the Townsend coefficient for the low partial pressure of isobutane. The maximum gain achieved for each of the gas mixtures tested corresponds to a detection threshold that is smaller than $2 \mathrm{keV} / \mathrm{pad}$.

\subsection{In-beam tests}

The ACTAR TPC demonstrator was further characterized using lowenergy beams of stable ions. Beams of ${ }^{58} \mathrm{Ni}$ and ${ }^{24} \mathrm{Mg}$ were produced at GANIL and were accelerated in the first separated sector cyclotron (CSS1) to energies of $4.0 \mathrm{MeV} / \mathrm{u}$ and $4.6 \mathrm{MeV} / \mathrm{u}$, respectively. The beams were then sent to the G3 experimental area where they passed through a thin (100 or $200 \mu \mathrm{g} / \mathrm{cm}^{2}$ ) carbon stripper foil and a set of two position-sensitive tracking detectors before traversing the Mylar foil entrance window of the detector.

The first test used the ${ }^{58} \mathrm{Ni}$ beam that had an average energy of $\sim 3.0 \mathrm{MeV} / \mathrm{u}$ after the Mylar entrance window. The detector was filled with 90 mbar of pure $\mathrm{iC}_{4} \mathrm{H}_{10}$ and the cathode and mesh voltages were set to $-1500 \mathrm{~V}$ and $-300 \mathrm{~V}$, respectively. With this beam energy, gas and gas pressure, the ${ }^{58} \mathrm{Ni}$ ions had a range of approximately $15 \mathrm{~cm}$ and thus were stopped in the active volume of the detector. The electron drift velocity was estimated using Magboltz [17] to be $2.5 \mathrm{~cm} / \mu \mathrm{s}$. The gain of the MICROMEGAS was deduced to be 70, which is in good agreement with the results obtained in Fig. 7b) for pure $\mathrm{iC}_{4} \mathrm{H}_{10}$. Based on the SRIM/TRIM calculations [22] shown in Fig. 8a), the energy losses of the ${ }^{58} \mathrm{Ni}$ beam, the ${ }^{12} \mathrm{C}$ ions and the scattered protons span nearly two orders of magnitude. To avoid saturating the signals on the 6 rows of pads directly under the beam axis, a lower gain of $1 \mathrm{pC}$ was 


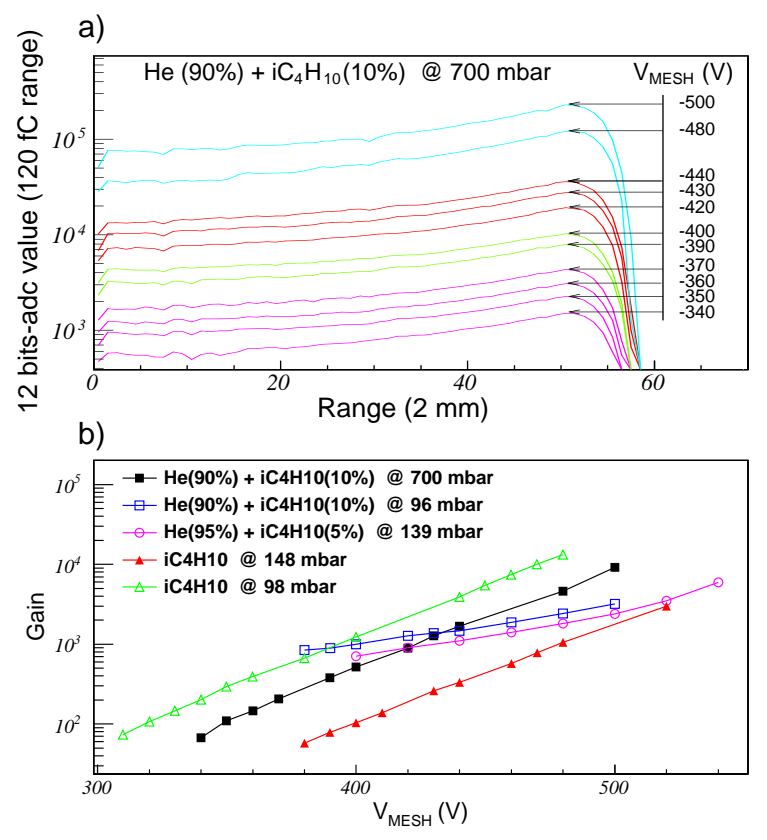

Figure 7: (Color Online) a) Average charge profiles of $5.8 \mathrm{MeV}{ }^{244} \mathrm{Cm}$ alpha particles obtained in a gas mixture of $\mathrm{He}(90 \%)+\mathrm{iC}_{4} \mathrm{H}_{10}(10 \%)$ at 700 mbar using several different voltages applied to the micromesh. The gain setting of the pad electronics is indicated by the color of the profiles: pink for $120 \mathrm{fC}$, green for $240 \mathrm{fC}$, red for $1 \mathrm{pC}$ and blue for 10 pC. b) Gain curves obtained for: $\mathrm{He}(90 \%)+\mathrm{iC}_{4} \mathrm{H}_{10}(10 \%)$ at 700 mbar (black solid squares), $\mathrm{He}(90 \%)+\mathrm{iC}_{4} \mathrm{H}_{10}(10 \%)$ at 96 mbar (blue open squares), $\mathrm{He}(95 \%)+\mathrm{iC}_{4} \mathrm{H}_{10}(5 \%)$ at 139 mbar (pink open circles), $\mathrm{iC}_{4} \mathrm{H}_{10}$ at 148 mbar (red solid triangles) and $\mathrm{iC}_{4} \mathrm{H}_{10}$ at 98 mbar (green open triangles).

applied to these channels. A higher gain of $120 \mathrm{fC}$ was used for the pads that were off beam axis. The geometry-dependent gain settings that were applied are shown in Fig. 8 8 ). In Fig. 8 8 ), the charge projection of a single event corresponding to the scattering of a proton in the gas by an incident ${ }^{58} \mathrm{Ni}$ beam ion is presented. In this figure, the ${ }^{58} \mathrm{Ni}$ ion is stopped in the chamber. The tail of the Bragg peak begins near column 42 and steadily decreases. At column 50, the tail extends past the $1 \mathrm{pC}$ low-gain region and reaches the $120 \mathrm{fC}$ high-gain region where the remaining signal is amplified.

Identification of the scattered particles was performed using the correlation between the reconstructed range of the particles in the chamber and the total charge collected on the pad plane. In Fig. 9a), this method is clearly able to distinguish between the scattered protons and the ${ }^{12} \mathrm{C}$ ions. After 

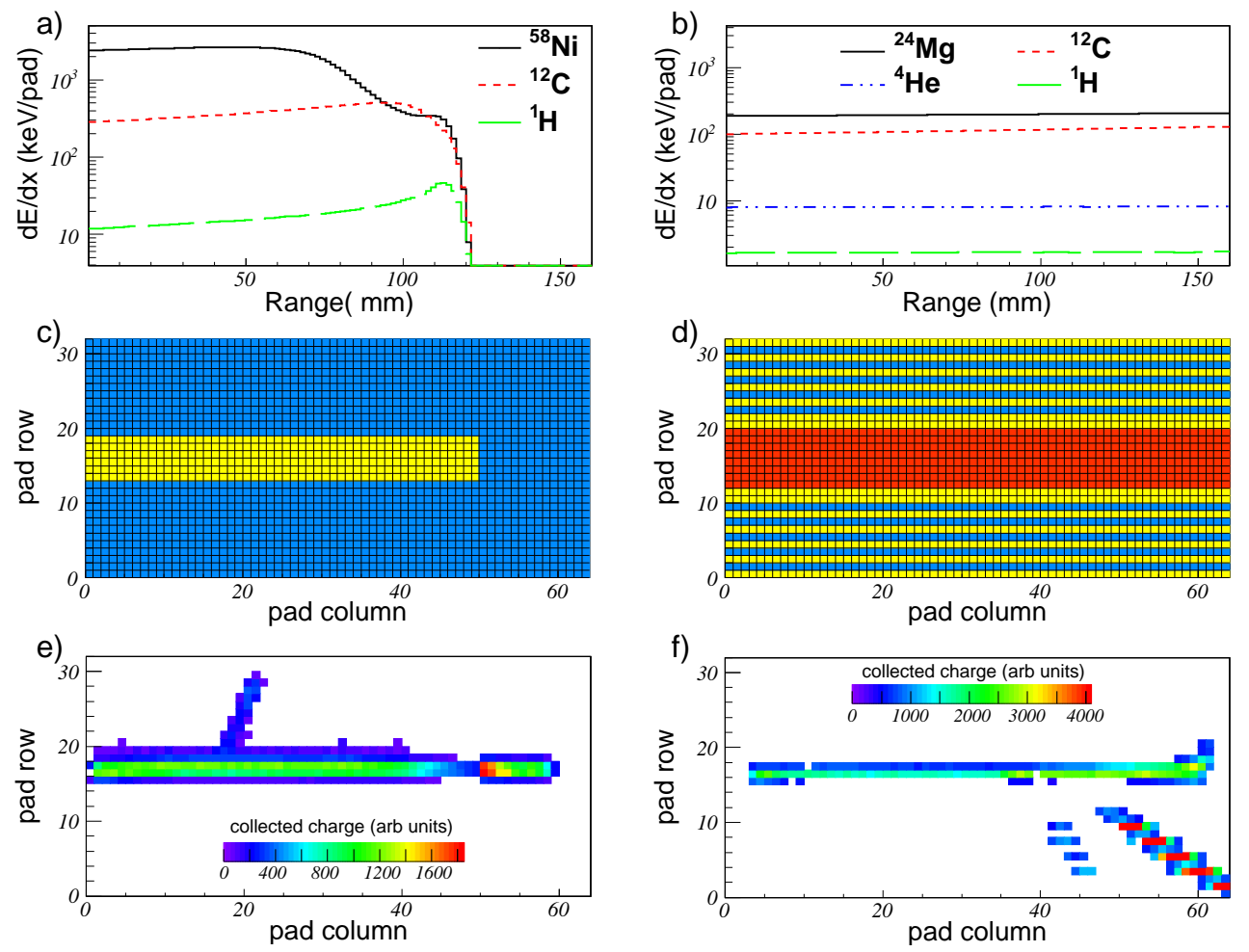

Figure 8: (Color Online) Top: SRIM energy-loss profiles of the particles involved in the ${ }^{58} \mathrm{Ni}$ test (a) and the ${ }^{24} \mathrm{Mg}$ test (b). Middle: Gain settings applied to the pad plane for the ${ }^{58} \mathrm{Ni}$ test (c) and the ${ }^{24} \mathrm{Mg}$ test (d). Blue indicates pads with $120 \mathrm{fC}$ range, yellow for $1 \mathrm{pC}$ range and red for $10 \mathrm{pC}$ range. Bottom: Charge projection of the scattering of a proton by a ${ }^{58} \mathrm{Ni}$ ion (e) and the scattering of a proton and ${ }^{4} \mathrm{He}$ by a ${ }^{24} \mathrm{Mg}$ ion (f).

selecting proton scattering events, the excitation energy spectrum of ${ }^{58} \mathrm{Ni}$ at center of mass angles between 30 and 100 degree was reconstructed using the correlation between the angle of the proton, its energy (determined from its range in the gas) and the reaction energy (from $1.7 \mathrm{~A}$ to about $0.2 \mathrm{~A} \mathrm{MeV}$ ) estimated from the position of the reaction vertex that is the intersection of the beam and proton trajectories. The resulting excitation energy spectrum is presented in Fig.9b), with in the inset, the center of mass angular domain and the reaction energy domain covered. The energy resolution obtained was $\sim 175 \mathrm{keV}$ (FWHM), which is excellent for these types of detectors. For com- 
parison, a recent result obtained with a similar prototype detector achieved a resolution of $800 \mathrm{keV}$ (FWHM) for the reconstructed excitation energy spectrum of ${ }^{6} \mathrm{He}[23]$.
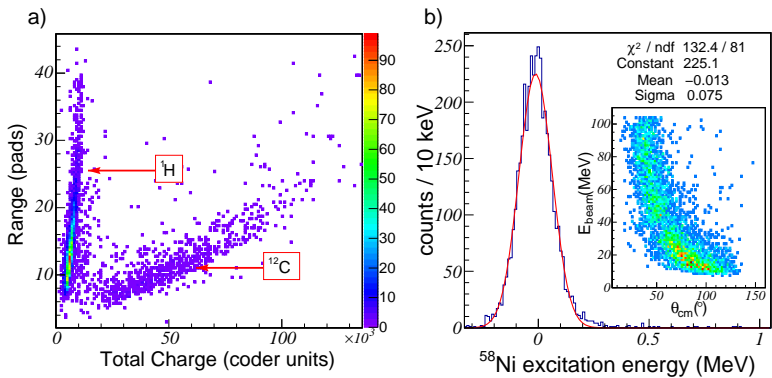

Figure 9: (Color Online) a) Particle identification plot obtained by correlating the range of the particles stopping in the active volume with the total charge deposit. Scattered protons and carbon ions are well separated. b) Excitation energy spectrum reconstructed for the ${ }^{58} \mathrm{Ni}+\mathrm{p}$ reaction. A Gaussian fit to this distribution (red line) results in an energy resolution of $\sim 175 \mathrm{keV}$ (FWHM). Inset) center of mass angular and reaction energy domain covered by the present analysis.

A second test was performed using a ${ }^{24} \mathrm{Mg}$ beam at $4.6 \mathrm{MeV} / \mathrm{u}$. After passing through the carbon stripper foil, the beam tracking detectors and the Mylar entrance window of the TPC, the ${ }^{24} \mathrm{Mg}$ beam energy was $\sim 4.0 \mathrm{MeV} / \mathrm{u}$. The demonstrator was filled with a mixture of $\mathrm{He}(90 \%)+\mathrm{iC}_{4} \mathrm{H}_{10}(10 \%)$ at 200 mbar. The cathode voltage was set to $-1900 \mathrm{~V}$ and the mesh voltage to $-380 \mathrm{~V}$. With these settings, the electron drift velocity was estimated to be $1.7 \mathrm{~cm} / \mu \mathrm{s}$ and the gain of the MICROMEGAS was approximately 500 (see Fig. (7). As shown in Fig $8 \mathrm{~b}$ ), the energy loss of the scattered ${ }^{12} \mathrm{C},{ }^{4} \mathrm{He}$ and ${ }^{1} \mathrm{H}$ ions span nearly two orders of magnitude. To be able to detect all 3 particles simultaneously with a transverse multiplicity of three pads to ensure a good track reconstruction, the electronics gain across the pad rows were alternated between high gain (120 fC) and low gain (1 pC). The central region directly under the beam axis was set to an even lower gain of $10 \mathrm{pC}$. The geometrical gain map employed is shown in Fig. 8d). A sample pile-up event is presented in Fig. 8f) and shows the charge projection that contains scattering events of both a proton and a ${ }^{4} \mathrm{He}$ ion. The proton track is only visible on the pads at $120 \mathrm{fC}$ gain and thus cannot be observed on the alternating $1 \mathrm{pC}$ rows. The track of the ${ }^{4} \mathrm{He}$ ion is clearly visible on the $1 \mathrm{pC}$ rows but its signals saturate the rows set to $120 \mathrm{fC}$ gain. The ${ }^{24} \mathrm{Mg}$ beam above the $10 \mathrm{pC}$ gain region is 
also observed without saturating the channels. By carefully optimizing the gain settings for the individual rows, this technique can be used to extend the effective dynamic range of the system. A second method to identify the scattered particles employed a set of silicon detectors that were placed on the sides of the chamber. A plot of the energy loss (total charge) of the particles in the active part of the TPC (obtained by summing the charges on alternating rows as appropriate) versus the energy recorded in the silicon detectors is presented in Fig. 10. The two distinct groups of events in this figure correspond to ${ }^{1} \mathrm{H}$ and ${ }^{4} \mathrm{He}$ ions.

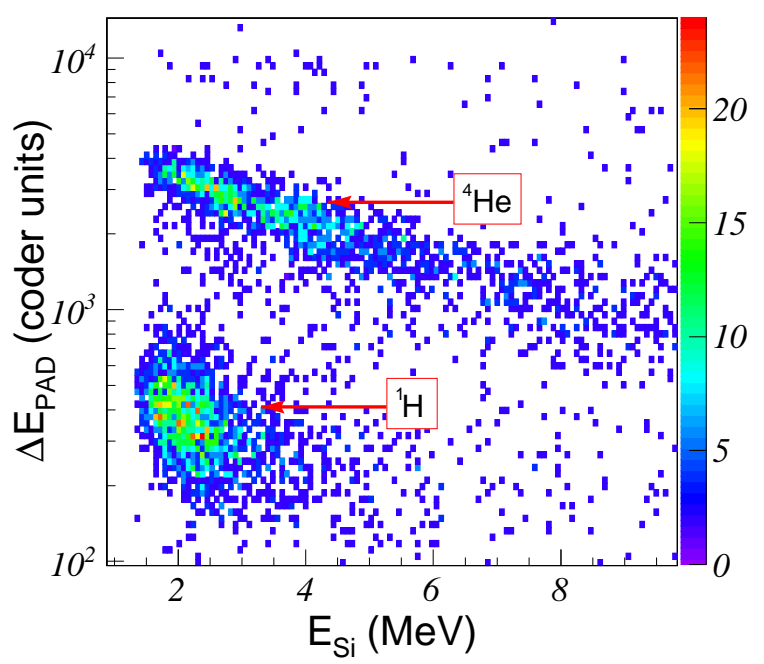

Figure 10: (Color Online) Particle identification for scattered ${ }^{1} \mathrm{H}$ and ${ }^{4} \mathrm{He}$ particles from a beam of ${ }^{24} \mathrm{Mg}$ ions at $4.0 \mathrm{MeV} / \mathrm{u}$. The energy deposited (charge) on the pad plane is plotted versus the energy recorded in a set of $\mathrm{Si}$ detectors located on the sides of the chamber (perpendicular to the beam axis).

Finally, the multi-particle tracking capabilities of the ACTAR TPC demonstrator coupled to the GET electronics were investigated during the beam tests. Some typical events recorded using both the ${ }^{58} \mathrm{Ni}$ and the ${ }^{24} \mathrm{Mg}$ beams are presented in Fig. 11. The voxel reconstruction of the system permits to resolve the pileup of events, as illustrated on the two 3D plots on the left, and to track multi-particle final states (more than two particles) as depicted on the plots at the right of the figure. 


\subsection{Summary and discussion}

Some of the key results that were obtained from the tests and calibrations described above are summarized and where applicable compared to results previously obtained from similar detectors.

Using the laser light calibration technique described above in Sec. 3.3, a horizontal angular resolution of $\pm 0.06^{\circ}$ (FWHM) was obtained. For a detector length of $128 \mathrm{~mm}$, this corresponds to position resolution of only $\pm 0.13 \mathrm{~mm}$ (FWHM). As straggling effects in the gas are absent with the laser light, this result can be considered to be the intrinsic angular resolution of the detector and depends primarily on the use of $2 \times 2 \mathrm{~mm}^{2}$ pixels. For particles with long trajectories, straggling effects will completely limit the angular resolution with typical results being on the order of $\pm 1^{\circ}$ in both the horizontal and vertical directions [12]. In terms of angular resolution, the use of larger pixels would therefore be expected to yield similar results. In the Maya active target that uses $8 \mathrm{~mm}$ long hexagonal pads, for example, angular resolutions of $\pm 1^{\circ}$ have also been obtained [20].

Energy resolutions obtained from summing the total charge on the pad plane were obtained using a mixed $3 \alpha$ source. The results obtained were typically $5 \%$ (FWHM) for $\alpha$ particles that deposited about $4 \mathrm{MeV}$ in the active volume of the detector. These results are in good agreement with our previous study that achieved 4 to $5 \%$ (FWHM) using a similar micromegas detector coupled to a $2 \times 2 \mathrm{~mm}^{2}$ pixelated pad plane [12]. These results are about a factor of 2 to 3 better than the value of $11 \%$ (FWHM) obtained in Ref. 24] from a MICROMEGAS segmented into one dimensional strips with a pitch of $5 \mathrm{~mm}$ and 13\% (FWHM) deduced in Ref. [23] from a similar pitch MICROMEGAS detector with a circular geometry. As described in Ref. [12], these results may not be fully comparable as they depend significantly on the details of the analysis procedures.

When tested in beam, an energy resolution of $175 \mathrm{keV}$ (FWHM) was obtained in Fig. 8 for the reconstructed excitation energy spectrum of elastically scattered ${ }^{58} \mathrm{Ni}$ ions by protons. This is about a factor 10 better than the $2 \mathrm{MeV}$ (FWHM) obtained for ${ }^{68} \mathrm{Ni}$ with the active target MAYA [25]. The improved energy resolution obtained in the present work compared to Refs. [23, 25] is presumably dominated by the overall range resolution obtained from alpha-particle source measurements.

In terms of overall gain and energy thresholds, several measurements obtained in a variety of different gases, mixtures and pressures were presented in Fig. 7b). These gain curves show that the detector can be operated up to a 
maximum gain of about $10^{4}$ (at $1 \mathrm{kHz}$ beam intensity), which corresponds to a low-energy threshold of approximately $0.15 \mathrm{keV} / \mathrm{pad}$, considering that a track can be properly treated if the transverse multiplicity is three pads. Through careful adjustments of the electronic gains on a channel-by-channel basis using the GET system, the effective dynamic range can be increased by an additional order of magnitude to approximately $10^{3}$. In the ${ }^{24} \mathrm{Mg}$ in-beam test described above, this feature was necessary to be able to observe scattered protons $(2 \mathrm{keV} / \mathrm{pad})$, alpha particles $(10 \mathrm{keV} / \mathrm{pad})$ and the ${ }^{24} \mathrm{Mg}$ ion beam (200 keV/pad) simultaneously and without saturating the electronics. The future version of ACTAR TPC will allow the polarization of the central pads located below the beam path in order to locally reduce the micromegas electric field and hence the gain, further increasing the dynamic range of the active target while limiting the risk of reaching the Raether limit, as shown in Ref. [23].

Particle identification and tracking capabilities of the detector were also explored in detail. Depending on the energies of the particles, a number of complementary measurements such as the energy loss (charge collected) in the target, the range of the particles in the gas and the energy deposited in auxiliary detectors can be combined to discriminate between particles with sufficient resolution. The use of a two dimensional segmented pad plane coupled with state-of-the-art electronics to digitize the electron arrival times on the pad plane are essential for studying reactions that lead to multiparticle final states (more than two particles) or to discriminate and remove pile up from the analysis as shown in Fig.11. Both of these will improve the overall efficiency and allow new studies to be performed that were not possible with previous active targets and TPCs in nuclear physics.

\section{Conclusion}

A novel 2048-channel prototype active target and time projection chamber for nuclear physics experiments was presented. The heart of the demonstrator features a $128 \mathrm{~mm} \times 64 \mathrm{~mm}$ pad plane that consists of a bulk MICROMEGAS, with a $220 \mu \mathrm{m}$ gap, mounted on a highly pixelated pad plane that features square pads with a pitch of $2 \times 2 \mathrm{~mm}^{2}$. An electric field cage based on planar wire grids surrounds the pads and encompasses the $17 \mathrm{~cm}$ height of the drift volume. In order to maintain a homogeneous drift electric field at the required level of $\leq 0.6 \%$, the use of a double wire cage was found to be essential. The use of wires rather than a solid structure is crucial 
for our applications as the field cage must be transparent to particles that escape the active volume of the detector. The double field cage design presented here, has achieved the homogeneity requirement while maintaining an optical transparency of $98 \%$.

Characterization and tests of the demonstrator were presented under a wide variety of conditions and with several different sources of ionizing radiation. These tests have provided many opportunities to test the electronics, to validate or improve upon the mechanical aspects of the design, to develop unique methods of calibration and to characterize the detector response with several different gases over a wide range of pressures. In terms of resolution, the horizontal angular resolution deduced using laser light was found to be $\sim 0.06^{\circ}$ (FWHM) while the energy resolution obtained from the total charge collected on the pad plane was deduced to be $\sim 5 \%$ (FWHM) for a $\sim 4 \mathrm{MeV}$ alpha particle. Tests performed with heavy ion beams of ${ }^{24} \mathrm{Mg}$ and ${ }^{58} \mathrm{Ni}$ have also clearly shown the particle identification and multi-particle tracking capabilities of such a detector. With more than 1 month of in-beam tests and now more than 4 years of intensive operation, the mechanical design has proven to be robust and reliable. All of these characteristics are extremely encouraging for the long-term use of such a detector for applications in nuclear physics with heavy-ion beams.

This prototype detector was designed and operated as a "demonstrator" for the much larger ACTAR TPC detection system that is presently being constructed. The new system will continue to use square pads of $2 \times 2 \mathrm{~mm}^{2}$ but the size of the pad plane will be increased to 16384 pads/channels. The design and experience gained with the demonstrator have thus played a crucial role in the development of ACTAR TPC. First experiments with this novel detection system are foreseen in 2018.

\section{Aknowledgements}

We would like to thank the PCB workshop at CERN and the electronics and the detector R\&D workshops of the Institut de Physique Nucléaire (IPN) from CNRS/IN2P3 in Orsay. The research leading to these results have received funding from the European Research Council under the European Unions Seventh Framework Program (FP7/2007- 2013)/ERC grant agreements $\mathrm{n}^{\circ} 335593$ and 617156. One of the authors, T.M., received funding from: Research Foundation - Flanders (FWO) under contract $n^{\circ} 133487$ and from the European Commission (EU-MSCA, MagicTin project) under con- 
tract $\mathrm{n}^{o}$ 661777. The research and development of the GET system was supported by the Agence Nationale de la Recherche (ANR) in France under contract $\mathrm{n}^{\circ}$ ANR-09-BLAN-0203-02 and the National Science Foundation (NSF) in the United States.

\section{References}

[1] G. D. Alkhazov et al., Phys. Rev. Lett. 78, 2313 (1997)

[2] Y. Mizoi et al., Nucl. Instrum. Meth. Phys. Res. A 431, 112 (1999)

[3] C. E. Demonchy et al., Nucl. Instrum. Meth. Phys. Res. A 583, 341 (2007)

[4] K. Miernik et al., Nucl. Instrum. Meth. Phys. Res. A 581, 194 (2007)

[5] B. Blank et al., Nucl. Instrum. Meth. Phys. Res. A 613, 65 (2010)

[6] S. Beceiro-Novo et al., Progress in Particle and Nuclear Physics 84, 124 (2015)

[7] Y. Giomataris et al., Nucl. Instrum. Meth. Phys. Res. A 376, 29 (1996)

[8] Y. Giomataris et al., Nucl. Instrum. Meth. Phys. Res. A 560, 405 (2006)

[9] F. Sauli, Nucl. Instrum. Meth. Phys. Res. A 386, 531 (1997)

[10] F. Sauli, Nucl. Instrum. Meth. Phys. Res. A 805, 2 (2016)

[11] W. Mittig et al., Nucl. Instrum. Meth. Phys. Res. A 784, 494 (2015)

[12] J. Pancin et al., Nucl. Instrum. Meth. Phys. Res. A 735, 532 (2014)

[13] E.C. Pollacco et al., To be submitted to Nucl. Instrum. Meth. Phys. Res. A

[14] G. Wittwer and F. Saillant and M. Blaizot and G. F. Grinyer and B. Raine and C. Belkhiria and S. Primault and C. Gueye, Real Time Conference RTC 2014 (2014)

[15] X. Grave and R. Canedo and J. F. Clavelin and S. Du and E. Legay, Real Time Conference RTC 2005 (2005) 
[16] B. Mauss and T. Roger and J. Pancin and S. Damoy and G.F. Grinyer, accepted for publication in EPJ Web of Conferences

[17] S.F. Biagi, Nucl. Instrum. Meth. Phys. Res. A 421, 234 (1999)

[18] E. Durand,Électrostatique Vols. 1 \& 2, Masson (1964)

[19] R. Veenhof, Garfield, http://garfield.web.cern.ch/garfield

[20] T. Roger et al., Nucl. Instrum. Meth. Phys. Res. A 638, 134 (2011)

[21] J. Giovinazzo et al., Nucl. Instrum. Meth. Phys. Res. A 840, 15 (2016)

[22] J. F. Ziegler and M. D. Ziegler and J. P. Biersack, Nucl. Instrum. Meth. Phys. Res. B 268, 1818 (2010)

[23] D. Suzuki et al., Nucl. Instrum. Meth. Phys. Res. A 691, 39 (2012)

[24] D. Suzuki et al., Nucl. Instrum. Meth. Phys. Res. A 660, 64 (2011)

[25] M. Vandebrouck et al., Phys. Rev. C 92, 024316 (2015) 

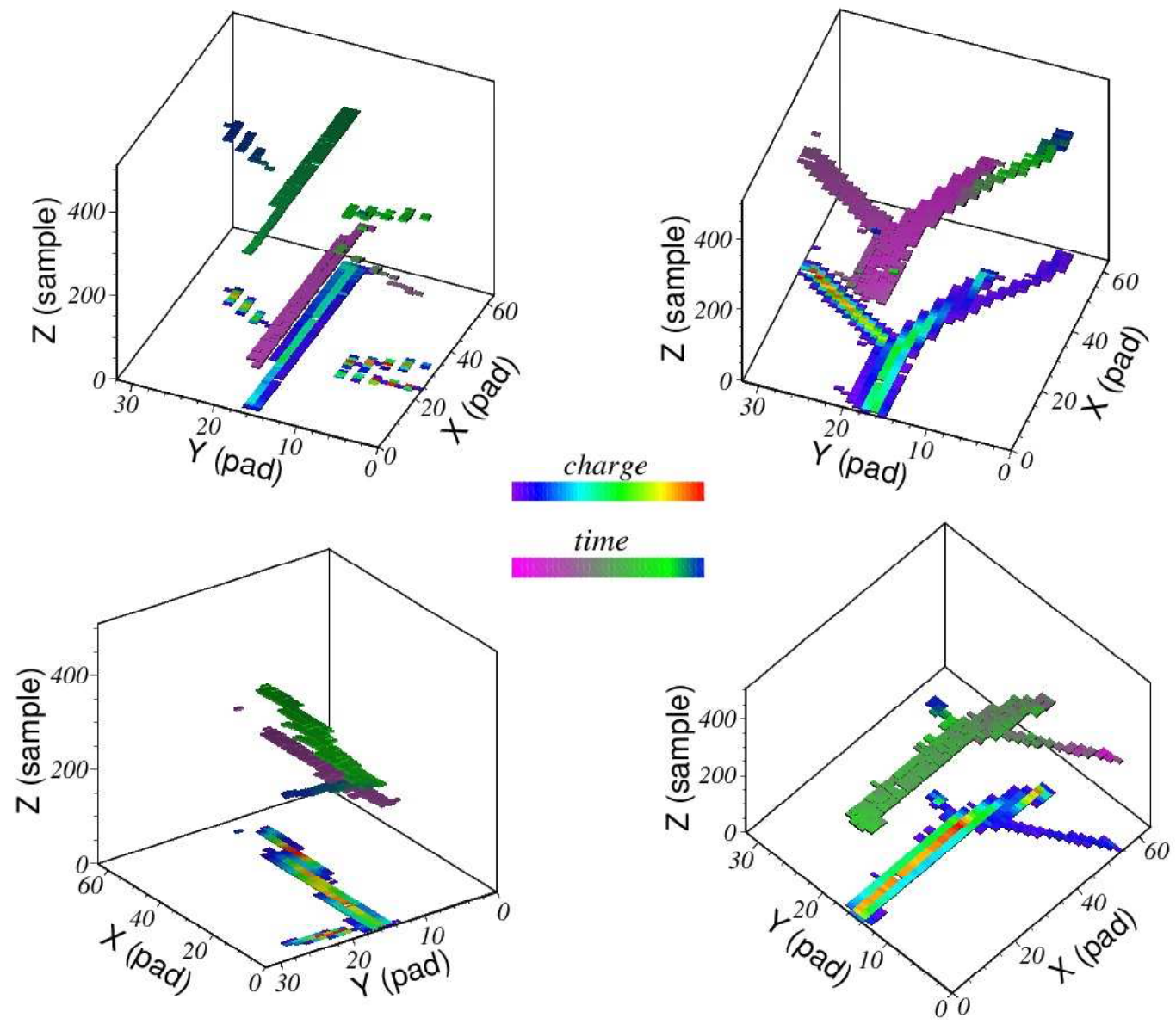

Figure 11: (Color Online) 3D events recorded by the ACTAR TPC demonstrator during the ${ }^{58} \mathrm{Ni}$ and the ${ }^{24} \mathrm{Mg}$ beam tests. The charge projection is plotted using a violet-to-red color palette, while the third dimension (time) is indicated on the voxels using a pink-toblue color palette. The left two plots show pileup events while the right two plots show multi-particle final state tracking capabilities. 